\title{
La regla proporcional para problemas de Bancarrota: Dos axiomatizaciones
}

Jony Alexander Rojas Rojas ${ }^{1}$ Roberto Carlos Picados Reyes ${ }^{2}$

\section{Información de artículo:}

Recibido: 30.06.2019

Aprobado: 30.10.2019

Palabras claves:

axioma de convexidad, problemas de bancarrota,

regla proporcional.

\section{Keywords:}

bankruptcy problems, convexity axiom, proportional rule.

\section{Resumen}

El principio de proporcionalidad es una de las formas de reparto más antiguas en la literatura de problemas de distribución. En este artículo se dan dos caracterizaciones de la regla proporcional: una como la única función que satisface los axiomas de aditividad, invarianza de escala, equidad y convexidad; y la otra caracterización usa los axiomas de tratamiento igualitario a iguales y convexidad.

\section{The proportional rule for Bankruptcy problems: Two axiomatizations}

\section{Abstract}

The principle of proportionality is one of the oldest forms of distribution in the literature of distribution problems. In this article two characterizations of the proportional rule are given: one as the only function that satisfies the axioms of additivity, invariance of scale, equity and convexity; and the other characterization uses the axioms of equal treatment to equals and convexity.

\footnotetext{
1 Doctor en Matemáticas Aplicadas, Profesor Investigador de la Universidad Nacional Autónoma de Nicaragua (UNANMANAGUA). Email: jonyrojas2o29@gmail.com.

${ }^{2}$ Licenciado en Ciencias de la Educación con Mención en Matemática, Asesor pedagógico nacional del Ministerio de Educación (MINED). Email: picador@mined.gob.ni.
} 


\section{Introducción}

Los problemas de bancarrota tratan en general con situaciones donde hay que distribuir un bien infinitamente divisible entre un grupo de agentes cuya demanda total sobrepasa la cantidad del bien disponible. Una manera natural de abordar estos problemas es la búsqueda de formas o reglas de repartición que satisfagan ciertas propiedades. Una de las reglas más antigua, comúnmente usada y ampliamente estudiada es la regla proporcional. Esta regla distribuye el bien proporcionalmente a los reclamos. O'Neill (1982), Aumann y Maschler (1985) y Curiel, Maschler y Tijs (1988) usan Teoría de juegos cooperativos como herramienta para definir reglas para problemas de bancarrota y analizar sus propiedades. La estrategia empleada es como sigue: a cada problema de bancarrota le asocian un juego cooperativo y a éste le aplican un concepto de solución (por ejemplo el concepto de solución de Shapley (1953)); dicho concepto de solución induce una regla para problemas de bancarrota. Aunque esta estrategia es muy efectiva, tiene la desventaja siguiente: Aumann y Maschler (1985) demuestran que si un problema de bancarrota es representado por un juego cooperativo, entonces es imposible obtener la regla proporcional de un concepto de solución que satisfaga las propiedades de simetría, eficiencia de Pareto e invarianza a equivalencia estratégica. Por esta razón, en este trabajo se adopta otra estrategia que caracterice la regla proporcional. A cada problema de bancarrota se asocia un conjunto convexo compacto apropiado que contenga el vector de reclamos. Usando que todo elemento de un conjunto convexo compacto de se escribe de manera única como combinación convexa de sus puntos extremos y los axiomas descritos en la sección 3, se obtienen dos caracterizaciones de la regla proporcional.

El trabajo está dividido en 5 secciones. La sección 2 contiene los conceptos y las definiciones necesarias para el desarrollo de las secciones que le preceden. En la sección 3 se presentan los axiomas y sus interpretaciones, y en la sección 4 los dos teoremas de caracterización. Por último, se dan las conclusiones y comentarios finales.

\section{Preliminares}

Primeramente, introduciremos un poco de notación. $\mathbb{Q}$ denota el conjunto de los racionales y $\mathbb{Q}_{+}$todos los racionales positivos. $\mathbb{R}$ y $\mathbb{R}_{+}$simbolizan el conjunto de los números reales y todos los números reales no negativos, respectivamente. $\mathbb{R}^{n}$ representa el conjunto de $n-u p l a s$ de números reales. Además, para todo $x, y \mathbb{R}^{n} y$ todo $\mu \in \mathbb{R}$,

$$
\mu x+y=\left(\mu x_{1}+y_{1} \ldots \mu x_{n}+y_{n}\right) .
$$

En este trabajo estudiamos situaciones donde un estado (dinero) E debe ser repartido entre un grupo de agentes (reclamantes) $N=\{1, \ldots, n\}, \operatorname{con} n>1 . c_{i}$ representa la parte del estado $E$ que reclama el agente $c \in N, c \in \mathbb{R}^{n}$ es el vector de reclamos 
$C=\sum_{i=1}^{n} c_{1}>E$ es el monto total de las reclamaciones. Una interrogante que surge de forma natural es: ¿Cómo dividir el estado entre los agentes? La respuesta a esta pregunta es revelada por medio de la definición de una regla. Una regla determina de manera única una distribución del estado entre los agentes. Más, formalmente:

Definición 1. Un problema de bancarrota es un par $(E, c) \in \mathbb{R}_{+} \times \mathbb{R}_{+}^{n}$ donde $c=\left(c_{1}, \ldots\right.$ $\left.c_{n}\right) y C>E$.

Los problemas de bancarrota son ampliamente estudiados en la literatura. Ver por ejemplo, O'Neill (1982) y Aumann y Maschler (1985), y para un escrutinio completo referirse a Thomson (2003), Thomson (2015) y Moulin (2002).

En lo que sigue supondremos que $c_{1} \leq c_{2} \leq \cdots \leq c_{n}$. La letra denotará el conjunto de todos los problemas de bancarrota y $C_{o}$ el conjunto de problemas bancarrota cuyos estados son racionales positivos, es decir,

$$
C=\left\{(E, c) / E \in \mathbb{R}+c \in \mathbb{R}^{n}+\right\} \text { y } C_{O}=\left\{(E, c) / E \in \mathbb{Q}+, c \in \mathbb{R}^{n}+\right\} .
$$

Definición 2. Una regla para problemas de bancarrota (o simplemente una regla) es una función $f: C \rightarrow \mathbb{R}^{n}$ que satisface lo siguiente: para todo $(E-c) \in C, \sum_{i=1}^{n} f_{i}(E, c)=E$ y $0 \leq f_{i}(E, c) \leq c_{i}$ para todo $i=1, \ldots, n$.

En la Definición 2, el número $f_{i}(E, c)$ es la parte que le corresponde al agente $i \in N$ del estado $E$ de acuerdo a la regla $f$. Se puede observar que bajo la asignación de una regla $\left(0 \leq f_{i}(E, c)\right)$ ningún agente tiene que pagar, todo agente recibe como máximo su monto reclamado $\left(f_{i}(E, c) \leq c_{i}\right)$ y se garantiza que el estado se distribuye en su totalidad $\left.\sum_{i=1}^{n} f_{i}(E, c)=E\right)$.

A continuación definimos la regla que se estudiará en este artículo.

Definición 3. La regla de reparto proporcional es la función $P: C \rightarrow \mathbb{R}^{n}$ tal que $P_{i}(E, c)=\lambda c_{i}, \lambda=\frac{E}{C}$ e $i=1, \ldots, n$.

Es decir, la regla proporcional distribuye el estado proporcionalmente a los reclamos.

El propósito de este artículo será mostrar que la regla proporcional dada por la Definición 3 está determinada de manera única por un cierto conjunto de propiedades, que se describen en la siguiente sección. 


\section{Axiomas}

Ahora, se define el conjunto de axiomas que requiere cumplir una regla en este trabajo. El primer axioma establece que si el estado se presenta en dos partes $E y E^{\prime}$, entonces la asignación de la regla al sumar las partes debe ser la suma de las asignaciones para cada una de ellas, siempre que el vector de reclamaciones se mantenga fijo. Es decir,

Aditividad. Para todo $(E, c),\left(E^{\prime}, c\right) \in C$

$$
f\left(E+E^{\prime}, c\right)=f(E, c)+f\left(E^{\prime}, c\right)
$$

Moulin (1987) y Chun (1988) utilizan esta propiedad en problemas de excedentes y asignación, respectivamente. El siguiente axioma trata con escala en el vector de reclamos, es decir, escalando las reclamaciones por una constante estrictamente positiva no afecta el resultado asignado por la regla.

Invarianza de escala. Para todo $(E, c) \in C$ y todo $\mu \in \mathbb{R}_{+}$tal que $\sum_{i=1}^{n} \mu c_{i}>E$

$$
f\left(E, \mu_{c}\right)=f(E, c)
$$

El próximo axioma dice que si dos agentes reclaman el mismo monto, entonces la regla debe asignar iguales cantidades para ambos agentes. Más precisamente,

Tratamiento igualitarioaiguales.Para todo $(E, c) \in C$ y todo $k, l \in N$ tal que $c_{k}=c_{l}$

$$
f_{k}(E, c)=f_{l}(E, c) \text {. }
$$

Considere un problema de bancarrota con la siguiente peculiaridad: $k \leq n$ agentes deciden condonar la deuda, es decir, cada uno reclama cero unidades y cada uno de los $n-k$ agentes restantes reclama una unidad. Además, el monto a dividir es una unidad, esto es, lo que hay para repartir es precisamente lo que los $n-k$ agentes reclaman. La próxima propiedad establece que para este tipo de problemas de bancarrota, la regla debe de asignar el estado de forma equitativa entre los $n-k$ agentes. Sin pérdida de generalidad: para $k=0,1, \ldots, n-1$, considere el vector de reclamos siguiente:

$$
e^{k}=(\underbrace{0, \ldots 0,}_{k} \underbrace{1, \ldots, 1}_{n-k}) \text {. }
$$

Equidad. Para todo $k \in\{0,1, \ldots n-2\}$ y todo $\left(1, e^{k}\right) \in C$ 


$$
f\left(1, e^{k}\right)=\frac{1}{n-k} e^{k}
$$

De acuerdo al axioma anterior, una regla equitativa da $f\left(1, e^{0}\right)=f(1, \underbrace{1, \ldots 1}_{n})=\frac{1}{n}$.

Notemos que el axioma de tratamiento igualitario a iguales implica el axioma de equidad, pero no al revés. Por otro lado, si $f$ es una regla para problemas de bancarrota, entonces $f\left(1, a \cdot e^{n-1}\right)=(\underbrace{0, \ldots 0}_{n-1} a)$ para todo $a \geq 1$.

Finalmente, la siguiente propiedad es introducida por Plata-Pérez, Sánchez-Pérez y Sánchez-Sánchez (2015) y fue utilizada para obtener una caracterización del índice de Gine. Nosotros adecuamos esta propiedad al contexto de problemas de bancarrota. Considere una pareja de problemas de bancarrota cuyos estados y montos totales de las reclamaciones coinciden, respectivamente. El axioma pide que la regla aplicada a la combinación convexa de tales problemas de bancarrota sea exactamente la combinación convexa de la regla de cada uno.

$$
\begin{gathered}
\text { Convexidad. Para todo }(E, c),\left(E, c^{\prime}\right) \in C, \operatorname{con} C=C^{\prime}, y \text { todo } \beta \in[0,1] \\
f\left[\beta(E, c)+(1-\beta)\left(E, c^{\prime}\right)\right]=f\left(E, \beta c+(1-\beta) c^{\prime}\right) \beta f(E, c)+(1-\beta) f\left(E, c^{\prime}\right) .
\end{gathered}
$$

\section{Resultados}

En esta sección se presentan dos caracterizaciones de la regla proporcional, una en $C_{0}$ y otra en el caso general $C$, por medio de los axiomas descritos en la sección anterior. Dichos resultados son los principales de este trabajo; para lograrlo necesitamos de algunos lemas que serán de utilidad en la caracterización de la regla proporcional.

La idea para mostrar que los axiomas propuestos caracterizan la regla proporcional parte de lo siguiente: para cada problema de bancarrota se define el conjunto convexo

$$
K(E, c)=\left\{x \in \mathbb{R}_{+}^{n} / \sum_{i=1}^{n} x_{i}=C, x_{1} \leq x_{2} \leq \cdots \leq x_{n}\right\}^{1} .
$$

Ahora, el lema siguiente muestra que todo elemento de $K(E, c)$ se expresa de manera única como combinación convexa de los vectores $\left\{z^{j}\right\}_{j=1}^{n}$, los cuales son los puntos extremos de $K(E, c)^{2}$ y se escriben como:

$$
z_{i}^{j}=\left\{\begin{array}{cc}
\frac{c}{n-j+1}, & \text { si } j \leq i \\
& \vdots: \\
0, & \text { en otro caso. }
\end{array}\right.
$$


Ejemplo 1. Para ilustrar el conjunto $K(E, c)$ y sus puntos extremos $z^{j}$, consideremos el problema de bancarrota $\left(\frac{5}{2}, 2,3,1\right)$, donde $E=\frac{5}{2} y c=(2,3,1)$. donde y. Por definición tenemos que:

$$
\begin{gathered}
K(E, c)=\left\{x \in \mathbb{R}_{+}^{3} / x_{1}+x_{2}+x_{3}=6, \quad x_{1} \leq x_{2} \leq x_{3}\right\} . \\
z^{1}=(2,2,2) \\
z^{2}=(0,3,3) \\
z^{3}=(0,0,6) .
\end{gathered}
$$

Notemos que cada $z^{j}$,con $j=1,2,3$, es un elemento de $K(E, c)$ y además ninguno de ellos está contenido en un segmento cuyos extremos estén en $K(E, c)$.

Lema 1. Sea $(E, c) \in C$. Para cada $y \in(E, c)$ existe una colección única de escalares $\left\{\alpha_{j}\right\}_{j=1}^{n}$, tal que $\sum_{i=1}^{n} \alpha_{i}=1$ y $y=\sum_{j=1}^{n} \alpha_{j} z^{j}$.

Además, los escalares están dados por:

$$
\alpha_{j}=\frac{(n-j+1)\left(y_{j}-y_{j-1}\right)}{C}
$$$$
\operatorname{con} j=1, \ldots, n \text { y } y_{0}=0 \text {. }
$$

Demostración. Considere la matriz de $n \times n, \quad A=\left(a_{i j}\right)$, definida por $a_{i j}=z_{i}^{i}$. Note que esta matriz siempre es invertible, y además, la ecuación lineal $y=\sum_{j=1}^{n} \alpha_{j} z^{j}$ es equivalente a la ecuación matricial $y=A \alpha$, donde $\alpha^{T}\left[\alpha_{1}, \ldots, \alpha_{n}\right]^{T}$ lo cual implica la existencia y unicidad de la colección de escalares $\left\{\alpha_{j}\right\}_{j=1}^{n}$.

Sea $y$ un elemento cualquiera de $K(E, c)$, entonces:

$$
\begin{aligned}
\sum_{j=1}^{n} \alpha_{j} z_{i}^{j} & =\sum_{j=1}^{i} \frac{(n-j+1)\left(y_{j}-y_{j-1}\right)}{C} \frac{C}{n-j+1}, \\
& =\sum_{j=1}^{i}\left(y_{j}-y_{j-1}\right), \\
& =y_{i} .
\end{aligned}
$$

Por otro lado, 


$$
\begin{aligned}
y_{i}-y_{i-1} & =\sum_{j=1}^{n} \alpha_{j} z_{i}^{j}-\sum_{j=1}^{n} \alpha_{j} z_{i-1}^{j}, \\
& =\sum_{j=1}^{i} \frac{\alpha_{j} C}{n-j+1}-\sum_{j=1}^{i-1} \frac{\alpha_{j} C}{n-j+1} \\
& =\frac{\alpha_{i} C}{n-i+1} .
\end{aligned}
$$

Luego, $\alpha_{i}=\frac{(n-i+1)\left(y_{i}-y_{i-1}\right.}{C}$ y es no negativo, porque $y \in K(E, c)$. Sólo nos resta probar que la suma de estos escalares es 1 :

$$
\begin{aligned}
\sum_{j=1}^{n} \alpha_{j} & =\sum_{j=1}^{n} \frac{(n-j+1)\left(y_{j}-y_{j-1}\right)}{C}, \\
& =\frac{1}{C}\left[\sum_{j=1}^{n}(n-j+1) y_{j}-\sum_{j=1}^{n-1}(n-j) y_{j}\right], \\
& =\frac{1}{C}\left[\sum_{j=1}^{n} y_{j}\right] \\
& =\frac{1}{C} C=1 .
\end{aligned}
$$

Lema 2. Sea $\left(E, c^{j}\right) \in C$, con $c^{j} \in K(E, c)$ para todo $j=1, \ldots, n$ y $\beta_{j} \geq 0$ tal que $\sum_{j=1}^{n} \beta_{j}=1$. Si $C \rightarrow \mathbb{R}^{n}$ es una regla que satisface el axioma de convexidad, entonces:

$$
f\left(\sum_{j=1}^{n}\left(E, \beta_{j} c^{j}\right)\right)=\sum_{j=1}^{n} \beta_{j} f\left(E, c^{j}\right) .
$$

Demostración. La prueba se hará por inducción sobre $n$. Para el caso $n=2$ la ecuación 3 es verdadera, ya que $\sum_{j=1}^{n} c^{j}=C$ y la regla $f$ satisface convexidad. Suponga que la ecuación 3 es verdadera para un entero $k>2$ :

$$
f\left(\sum_{j=1}^{k}\left(E, \beta_{j} c^{j}\right)\right)=\sum_{j=1}^{k} \beta_{j} f\left(E, c^{j}\right),
$$

donde $\left(E, c^{j}\right) \in \mathcal{C}, c^{j} \in K(E, c) \quad$ para todo $j=1, \ldots, k$ y $\beta_{j} \geq 0$ es tal que $\sum_{j=1}^{n} \beta_{j}=1$.

Ahora, considere $\left(E, c^{j}\right) \in \mathcal{C}, c^{j} \in K(E, c)$ para todo $j=1, \ldots, k+1$ y $\gamma_{j} \geq 0$ es tal que

$\sum_{j=1}^{n} \gamma_{j}=1$ 
Dado que $f$ satisface el axioma de convexidad,

$$
\begin{aligned}
f\left(\sum_{j=1}^{k+1} \gamma_{j}\left(E, c^{j}\right)\right) & =f\left(\sum_{j=1}^{k} \gamma_{j}\left(E, c^{j}\right)+\gamma_{k+1}\left(E, c^{k+1}\right)\right) \\
& =f\left(\left(1-\gamma_{k+1}\right) \sum_{j=1}^{k} \frac{\gamma_{j}}{1-\gamma_{k+1}}\left(E, c^{j}\right)+\gamma_{k+1}\left(E, c^{k+1}\right)\right), \\
& =\left(1-\gamma_{k+1}\right) f\left(\sum_{i=1}^{k} \frac{\gamma_{j}}{1-\gamma_{k+1}}\left(E, c^{j}\right)\right)+\gamma_{k+1}\left(E, c^{k+1}\right) .
\end{aligned}
$$

Claramente $\frac{\gamma_{j}}{1-\gamma_{k+1}} \geq 0$ y $\sum_{j=1}^{k} \frac{\gamma_{j}}{1-\gamma_{k+1}}=1$. Luego, por hipótesis inductiva se obtiene que

$$
\begin{aligned}
f\left(\sum_{j=1}^{k+1} \gamma_{j}\left(E, c^{j}\right)\right) & =\left(1-\gamma_{k+1}\right) \sum_{j=1}^{k} \frac{\gamma_{j}}{1-\gamma_{k+1}} f\left(E, c^{j}\right)+\gamma_{k+1}\left(E, c^{k+1}\right), \\
& =\sum_{j=1}^{k+1} \gamma_{j} f\left(E, c^{j}\right)
\end{aligned}
$$

Después de establecer los resultados anteriores, estamos listos para enunciar el primer resultado de este trabajo.

Teorema 1. Sea $f: C_{0} \rightarrow \mathbb{R}^{n}$ una regla para problemas de bancarrota. Entonces, es igual a la regla proporcional si y sólo si ésta satisface los axiomas de aditividad, invarianza de escala, equidad y convexidad.

Demostración. Primeramente, probaremos que si $f$ es igual a la regla proporcional, entonces satisface los axiomas de aditividad, invarianza de escala, equidad y convexidad.

Aditividad: Sean $(E, c),\left(E^{\prime}, c\right) \in C_{0}$. 


$$
\begin{aligned}
f_{i}\left(E+E^{\prime}, c\right) & =P_{i}\left(E+E^{\prime}, c\right) \\
& =\frac{\left(E+E^{\prime}\right)}{C} c_{i} \\
& =\left(\frac{E}{C}+\frac{E^{\prime}}{C}\right) c_{i} \\
& =\frac{E}{C} c_{i}+\frac{E^{\prime}}{C} c_{i} \\
& =P_{i}(E, c)+P_{i}\left(E^{\prime}, c\right) \\
& =f_{i}(E, c)+f_{i}\left(E^{\prime}, c\right) .
\end{aligned}
$$

Invarianza de escala: Sean $(E, c) \in \mathcal{C}_{0}$ y $\mu \in \mathbb{R}_{+}$tal que $\sum_{i=1}^{n} \mu c_{i}>E$.

$$
\begin{aligned}
f_{i}(E, \mu c) & =P_{i}(E, \mu c) \\
& =\frac{E}{\sum_{i=1}^{n} \mu c_{i}} \mu c_{i} \\
& =\frac{E}{\mu\left(\sum_{i=1}^{n} c_{i}\right)} \mu c_{i} \\
& =\frac{E}{C} c_{i} \\
& =P_{i}(E, c) \\
& =f_{i}(E, c) .
\end{aligned}
$$

Equidad: Sea $k \in\{0,1, \ldots n-2\}$ y $\left(1, e^{k}\right) \in \mathcal{C}_{0}$

$$
\begin{aligned}
& f_{i}\left(1, e^{k}\right)=P_{i}\left(1, e^{k}\right) \\
& =\left\{\begin{array}{c}
0 \text { si } i=1, \ldots, k \\
\frac{1}{n-k} \text { si } i=k+1, \ldots, n,
\end{array}\right.
\end{aligned}
$$

lo cual implica que $f\left(1, e^{k}\right)=P\left(, 1, e^{k}\right)=\frac{1}{n-k} e^{k}$. 
Convexidad: Consideremos $(E, C),\left(E, c^{\prime}\right) \in \mathcal{C}_{0}$, con $C=C^{\prime}$, y $\beta \in[0,1]$.

Los casos $\beta=0$ y $\beta=1$ no los consideramos, porque son evidentes. Sea $0<\beta<$ 1.

$$
\begin{aligned}
f_{i}\left[\beta(E, c)+(1-\beta)\left(E, c^{\prime}\right)\right] & =f_{i}\left(E, \beta c+(1-\beta) c^{\prime}\right) \\
& =P_{i}\left(E, \beta c+(1-\beta) c^{\prime}\right) \\
& =\frac{E}{\sum_{i=1}^{n}\left[\beta c_{i}+(1-\beta) c_{i}^{\prime}\right]}\left(\beta c_{i}+(1-\beta) c_{i}^{\prime}\right)
\end{aligned}
$$

Como $C=C^{\prime}$, entonces $\sum_{i=1}^{n}\left[\beta c_{i}+(1-\beta) C^{\prime}{ }_{i}\right]=C$. Luego,

$$
\begin{aligned}
\frac{E}{\sum_{i=1}^{n} \beta c_{i}+(1-\beta) c_{i}^{\prime}}\left(\beta c_{i}+(1-\beta) c_{i}^{\prime}\right) & =\frac{E}{C}\left(\beta c_{i}+(1-\beta) c_{i}^{\prime}\right) \\
& =\beta \frac{E}{C} c_{i}+(1-\beta) \frac{E}{C} c^{\prime}{ }_{i} \\
& =\beta P_{i}(E, c)+(1-\beta) P_{i}\left(E, c^{\prime}\right) \\
& =\beta f_{i}(E, c)+(1-\beta) f_{i}\left(E, c^{\prime}\right) .
\end{aligned}
$$

Ahora, supongamos que la regla $f: C_{0} \rightarrow \mathbb{R}^{n}$ satisface los axiomas de aditividad, invarianza de escala, equidad y convexidad. Sea $(E, c) \in C_{0}$ un problema de bancarrota arbitrario, pero fijo. Entonces existen números naturales $m$ y $n$ con $n \neq 0$ tal que $E=\frac{m}{n}$. Así,

$$
f(E, c)=f\left(\frac{m}{n}, c\right)=f(\underbrace{\frac{1}{n}+\cdots+\frac{1}{n}, c}_{m-\text { veces }})
$$

Por aplicación de aditividad en el lado derecho de la igualdad anterior, obtenemos

Por actividad y que $f(1, c)=f(\underbrace{\frac{1}{n}+\cdots+\frac{1}{n}}_{n-\text { vesces }}, c)$, entonces $f\left(\frac{1}{n}, c\right)=\frac{1}{n} f(1, c)$. Por tanto

$$
f(E, c)=m f\left(\frac{1}{n}, c\right) \text {. }
$$

$$
f(E, c)=m f\left(\frac{1}{n}, c\right)=\frac{m}{n} f(1, c)=E f(1, c) .
$$

Dado que $c \in K(E, c)$, entonces 


$$
c=\sum_{j=1}^{n} \alpha_{j} z^{j}, \operatorname{con} \alpha_{j}=\frac{(n-j+1)\left(c_{j}-c_{j-1}\right)}{C} .
$$

Sustituyendo la ecuación 5 en 4 se obtiene $f(E, c)=E f(1, c)=E f\left(1, \sum_{j=1}^{n} \alpha_{j} z^{j}\right)$. Como $f$ satisface convexidad e invarianza de escala,

$$
\begin{aligned}
f(E, c) & =E \sum_{j=1}^{n} \alpha_{j} f\left(1, z^{j}\right), \\
& =E \sum_{j=0}^{n-1} \alpha_{j+1} f\left(1, e^{j}\right) .
\end{aligned}
$$

Por el axioma de equidad y que $f$ es una regla se tiene,

$$
f(E, c)=E \sum_{j=0}^{n-1} \alpha_{j+1} \frac{1}{n-j} e^{j},
$$

Es decir,

$$
\begin{aligned}
f(E, c) & =E \sum_{j=0}^{n-1} \alpha_{j+1} \frac{1}{n-j} e^{j}, \\
& =E \sum_{j=0}^{n-1} \frac{(n-j-1+1)\left(c_{j+1}-c_{j}\right)}{C} \frac{1}{n-j} e^{j}, \\
& =\frac{E}{C} \sum_{j=0}^{n-1}\left(c_{j+1}-c_{j}\right) e^{j}, \\
& =\frac{E}{C} c .
\end{aligned}
$$

Observación 1. Note que los axiomas de invarianza de escala y equidad determinan una regla en los problemas de bancarrota cuyas reclamaciones sean los puntos extremos de $K(E, c)$ con $(E, c) \in C_{0}$, mientras que aditividad y convexidad determinan la regla para cualquier problema de bancarrota en $C_{0}$.

Teorema 2. Sea $f: C \rightarrow \mathbb{R}^{n}$ una regla para problemas de bancarrota. Entonces, $f$ es igual a la regla proporcional si y sólo si ésta satisface los axiomas de tratamiento igualitario a iguales y convexidad. 
Demostración. En el Teorema 1 se demostró que si $f$ es la regla proporcional, entonces $f$ satisface el axioma de convexidad. Ahora, veremos que si $f$ es igual a la regla proporcional, entonces satisface el axioma de tratamiento igualitario a iguales.

$$
\begin{aligned}
& \text { Sean }(E, c) \in \mathcal{C} \text { y } k, l \in N \text { tal que } c_{k}=c_{l}, \\
& \qquad f_{k}(E, c)=P_{k}(E, c)=\frac{E}{C} c_{k}=\frac{E}{C} c_{l}=f_{l}(E, c) .
\end{aligned}
$$

Suponga que la regla $f: \mathcal{C} \rightarrow \mathbb{R}^{n}$ satisface los axiomas de tratamiento igualitario a iguales y convexidad. Sea $(E, c) \in \mathcal{C}$ un problema de bancarrota arbitrario, pero fijo. Entonces por convexidad

$$
\begin{aligned}
f(E, c) & =f\left(E, \sum_{j=1}^{n} \alpha_{j} z^{j}\right), \\
& =\sum_{j=1}^{n} \alpha_{j} f\left(E, z^{j}\right) .
\end{aligned}
$$

Note que si $k, i \in N$ con $z_{i}^{j}, z_{k}^{j} \neq 0$, entonces $z_{i}^{j}=z_{k}^{j}$. Luego, por el axioma de tratamiento igualitario a iguales y que $f$ es una regla para problemas de bancarrota se tiene

$$
f(E, c)=\sum_{j=0}^{n-1} \alpha_{j+1} \frac{E}{n-j} e^{j}
$$

Luego,

$$
\begin{aligned}
f(E, c) & =\sum_{j=0}^{n-1} \alpha_{j+1} \frac{E}{n-j} e^{j} \\
& =\sum_{j=0}^{n-1} \frac{(n-j-1+1)\left(c_{j+1}-c_{j}\right)}{C} \frac{E}{n-j} e^{j} \\
& =\frac{E}{C} \sum_{j=0}^{n-1}\left(c_{j+1}-c_{j}\right) e^{j} \\
& =\frac{E}{C} c
\end{aligned}
$$


Ejemplo 2. Como una ilustración de los resultados anteriores (usando la misma notación), calculamos la asignación de la regla proporcional para el problema de bancarrota $(5,2,3,5)$, donde el estado $E=5$ y el vector de reclamaciones $c=(2,3,5)$.

Para el problema de bancarrota $(5,2,3,5)$,

y así

$$
\begin{aligned}
c & =(2,3,5)=\frac{3}{5} z^{1}+\frac{1}{5} z^{2}+\frac{1}{5} z^{3} \\
& =\frac{3}{5}\left(\frac{10}{3}, \frac{10}{3}, \frac{10}{3}\right)+\frac{1}{5}(0,5,5)+\frac{1}{5}(0,0,10)
\end{aligned}
$$

$$
\begin{aligned}
P(5,2,3,5) & =5 P(1,2,3,5) \\
& =5 P\left(1, \frac{3}{5}\left(\frac{10}{3}, \frac{10}{3}, \frac{10}{3}\right)+\frac{1}{5}(0,5,5)+\frac{1}{5}(0,0,10)\right) \\
& =5\left[\frac{3}{5} P\left(1, \frac{10}{3}, \frac{10}{3}, \frac{10}{3}\right)+\frac{1}{5} P(1,0,5,5)+\frac{1}{5} P(1,0,0,10)\right], \\
& =5\left[\frac{3}{5} P(1,1,1,1)+\frac{1}{5} P(1,0,1,1)+\frac{1}{5} P(1,0,0,1)\right] \\
& =5\left[\frac{3}{5} \times \frac{1}{3}(1,1,1)+\frac{1}{5} \times \frac{1}{2}(0,1,1)+\frac{1}{5} \times \frac{1}{1}(0,0,1)\right] \\
& =5\left(\frac{1}{5}, \frac{3}{10}, \frac{1}{2}\right) \\
& =\left(1, \frac{3}{2}, \frac{5}{2}\right)
\end{aligned}
$$

Ahora, por el Teorema 2, se tiene

$$
\begin{aligned}
P(5,2,3,5) & =P\left(5, \frac{3}{5}\left(\frac{10}{3}, \frac{10}{3}, \frac{10}{3}\right)+\frac{1}{5}(0,5,5)+\frac{1}{5}(0,0,10)\right), \\
& =\frac{3}{5} P\left(5, \frac{10}{3}, \frac{10}{3}, \frac{10}{3}\right)+\frac{1}{5} P(5,0,5,5)+\frac{1}{5} P(5,0,0,10), \\
& =\frac{3}{5} \times \frac{5}{3}(1,1,1)+\frac{1}{5} \times \frac{5}{2}(0,1,1)+\frac{1}{5} \times 5(0,0,1), \\
& =\left(1, \frac{3}{2}, \frac{5}{2}\right) .
\end{aligned}
$$




\section{Discusión y conclusiones}

Primeramente, describiremos brevemente el método usado en este trabajo para la caracterización de la regla proporcional. La idea es simple y consistió en descomponer un problema de bancarrota en subproblemas fáciles de resolver, luego la solución es la suma ponderada de las soluciones de los subproblemas de bancarrota. Además, dicho método dió como resultado dos caracterizaciones de la regla proporcional. La primera caracterización es en un subconjunto infinito del conjunto de todos los problemas de bancarrota y usa los axiomas de aditividad, invarianza de escala, equidad y convexidad; y la segunda fue en todo el conjunto de problemas por medio de los axiomas tratamiento igualitario a iguales y convexidad. Cabe señalar que auque el axioma de tratamiento igualitario a iguales y la condición de que ningún agente recibe un monto menor que cero implican el axioma de equidad, el regreso no se cumple.

Bergantiños y Vidal (2004) demuestran que no existe una regla de bancarrota que satisfaga el axioma de aditividad, siempre que las reclamaciones y el estado sean números naturales. Sin embargo, dos años depués estos mismos autores (ver Bergantiños y Vidal (2006)) prueban que la regla proporcional es la única que satisface el axioma de aditividad, siempre que $C \geq E$. Dado que por la Definición 2 en todo problema de bancarrota $(E, c)$ se satisface que $C>E$, el resultado de Bergantiños y Vidal (2006) no se aplica. Además, el espacio de problemas con que ellos trabajan contiene como subconjunto propio el espacio de problemas que consideramos. Dos años más tarde Marchant (2008) prueba que la regla proporcional es la única que satisface el axioma de invarianza multiplicativa, es decir, $f(\gamma E, c)=f(E, c)$, siempre que $\sum_{i=1}^{n} \gamma c_{i} \geq C$, entonces $f(E, c)=P(E, c)$. De nuevo, al igual que en Bergantiños y Vidal (2008), el espacio de problemas de bancarrota que considera Marchant contiene como subconjunto propio a $C$ por esta razón y porque el axioma de invarianza usado en nuestro trabajo es más restrictivo $\left(\sum_{i=1}^{n} \gamma c_{i}>C\right)$ se deduce que el resultado de Marchant (2008) no es válido en el espacio de problemas que aquí se considera.

Por otro lado, estamos convencidos (al igual que Biembengut y Hein, 2004); (Burkhardt y Hein, 2006); (Betancur, Montoya, Mesa, y Villa, 2013) que la modelación matemática es una estrategia poderosa para incentivar, afirmar y comprender la matemática como una herramienta indispensable en la compresión de los fenómenos naturales y sociales, más precisamente el fenómeno de la escasez presente en esta investigación.

La proporcionalidad es un tema recurrente en el curriculum nacional de matemática, tanto en primaria, secundaria y a nivel universitario. El tema de la proporcionalidada tiene su génesis en primaria con el estudio de las razones en quinto grado, continuando en sexto con la definición de proporción mediante el uso de razones. En secundaria se hace más fino el estudio de la proporcionalidad con la insercción 
en séptimo grado de la quinta unidad llamada "proporcionalidad", aquí se aborda la proporcionalidad directa e inversa.

A nivel Universitario, la UNAN-Managua tiene instaurado el estudio de la proporcionalida en "Matemática General", la cual corresponde al área de formación general de la mayoría de las carreras ofertadas por la UNAN -Managua.

Teniendo como referencia el párrafo anterior creemos que esta investigación, donde se modeló matemáticamente un fenómeno de la escasez (la bancarrota) y se dió solución mediante un concepto matemático ampliamente estudiado (la proporcionalidad) en el curruculum nicaragüense, puede ayudar a mejorar el interes de los dicentes por la matemática y dar respuesta a la pregunta que todo matemático alguna vez ha escuchado ¿Para qué sirven las matemáticas?

\section{Lista de referencias}

Aumann, R.J. y Maschler, M. (1985). Game theoretic analysis of a bankruptcy problem from the Talmud. Journal of Economy Theory, (36): 195- 213.

Betancur, J., Montoya, J., Mesa, L., \& Villa, J. (2013). La modelación matemática: un ejemplo en el contexto cafetero. Revista Científica, 474 - 478.

Biembengut, M., \& Hein, N. (2004). Modelación matemática y los desafíos para enseñar matemática. Educación matemática, vol.16, 105-125.

Biembengut, M., \& Hein, N. (2006). Modelaje matemático como método de investigación en clasesde matemáticas. $\mathrm{V}$ festival internacional de matemática. De Costa a Costa (págs. 1 - 25). Costa Rica: Educación Matemática.

Bergantiños, G. y Vidal-Puga, J. (2004). Additive rules in bankruptcy problems and in other related problems. Mathematical Social Sciences, (47): 87-101.

Bergantiños, G. y Vidal-Puga, J. (2006). Additive rules in discrete allocation problems. European J. Oper. Res., (172): 971-978.

Chun, Y. (1988). The proportional solution for rights problems. Mathematical Social Sciences, (15): 231- 246.

Curiel I.J., Maschler, M. y Tijs, S.H. (1988). Bankruptcy games. Z. Op. Res, (31): A143-A159.

Ministerio de Educación. (2015). Matemática 5to grado Educación primaria. Managua, Nicaragua: MINED. 
Ministerio de Educación. (2015). Matemática 6to grado Educación primaria. Managua, Nicaragua: MINED.

Ministerio de Educación. (2015). Matemática $7 m o$ grado Educación Secundaria. Managua, Nicaragua: MINED.

Marchant, T. (2008). Scale invariance and similar invariance conditions for bankruptcy problems. Social Choice and Welfare, (31): 693-707. Erratum in Social Choice and Welfare, (31): 709-710.

Moulin, H. (1987). Equal or proportional division of a surplus, and other methods. International Journal of Game Theory, (16): 161- 186.

Moulin, H., (2002). Axiomatic cost and surplus sharing. En Arrow, K., Sen, A. y Suzumura, K. (Eds.), Handbook of Social Choice and Welfare. (pp. 289- 357). Elsevier: Amsterdam.

O'Neill, B. (1982). A problem of rights arbitration from the Talmud. Mathematical Social Sciences, (2): 345-371.

Plata-Pérez L., Sánchez-Pérez J. y Sánchez-Sánchez F. (2015). An elementary characterization of the Gini index. Mathematical Social Sciences, (74): 79-83

Shapley L. S. (1953). A value for n-person games. En H. W. Kuhn and A. W. Tucker, editors, Contributions to the Theory of Games, volume II of Annals of Mathematics Studies. (pp. 307-317). Princeton University Press, Princeton.

Thomson, W. (2003). Axiomatic and game-theoretic analysis of bankruptcy and taxation problems: a survey. Mathematical Social Sciences, (45): 249- 297.

Thomson, W. (2015). Axiomatic and game-theoretic analysis of bankruptcy and taxation problems- An update. Mathematical Social Sciences, (74): 41-59.

Universidad Nacional Autónoma de Nicaragua, Managua (2013). Plan de Estudios de todas las Carreras, Facultad de Educación e Idiomas. Managua: UNAN- Managua: Autor 